\title{
DYEING BEHAVIOR AND PERFORMANCE OF PREPARED BENZIMIDAZOLE AND CHROMENE DISPERSE DYES AS APPLIED ON POLYESTER FIBRE, AND ITS BLENDS WITH WOOL.
}

S. SHAKRA*, M. S. ABOUL-FETOUH , A.-B. GHATTAS**,and F. EL SAYED HASSAN*.

Chemistry Department, Faculty of Science, Al-Azhar University, Nasr City, Cairo , Egypt.

* Textile Research Division, National Research Centre, Dokki, Cairo, Egypt .

**Chemistry Department, Faculty of Science, Sohag University, Egypt.

\begin{abstract}
Azo disperse dyes Ia-f - Va-f were synthesized from corresponding amines, where IR and visible spectra were evaluated. The prepared dyes were used for dyeing polyester, polyester woolen type and wool fibres. A range of bright and leveled shade have been obtained on application of these disperse dyes. The K/S values for various dyed substrate are in the following order, Wool > polyester/wool > polyester.
\end{abstract}

\section{Introduction}

Dyes based on heterocyclic Compounds as azo Components tend mostly to produce brilliant shade high tinctorial strength and hue ranging from yellow to dark brown $^{(1)}$.

Disperse dyes have been applied to a large number and variety of fibres, such as: cellulose acetate, triacetate, polyamide, and polyester fibres. Disperse dyes have therefore achieved an importance which is probably greater than the suggested weights used. These were the first type to be applied directly to fibre from an insoluble suspension. Their preparation for dyeing included a process adopting particle size reduction, and often dyeing in the presence of dispersing agent. The mechanism of dyeing with them would assume initially their passing from suspension through solution onto the fibre to which they become attached by nonpolar forces. ${ }^{(2)}$.

In recent years, the introductions of man-made and synthetic fibres have accelerated the development of new application methods, therefore assisting the dyer to utilize such more complex dyes. 


\section{Experimental}

\section{Synthesis of dyestuffs:}

1) Synthesis of 5-aminobenzimidazole derivatives (A, B\&C) ${ }^{(3-6)}$ (General procedure).

A suspension of 5-nitrobezimidazole derivative $(0.048 \mathrm{~mol})$ in acetic acid (300 $\mathrm{ml}, 20 \%)$ was stirred at $60 \mathrm{C}^{\circ}$ while iron powder (20gm) was added gradually. After $2 \mathrm{hrs}$. the solution was cooled, diluted with water and the formed precipitate was filtered off, the product (5-aminobenzimidazole, A; 5-amino-2methylbenzimidazole, B and 5-amino-2-trifluoromethylbenzimidazole, C) was extracted from the precipitate by acetone. After evaporation of acetone the product was crystallized from ethanol.

(Compound A, yield, 80\%, m.p., $113 \mathrm{c}^{\mathrm{o}}$; Compound B, yield, 88\%, m.p., 229$231 \mathrm{c}^{\mathrm{o}}$ and Compound C, yield, 68\%, m.p., 185-189 $\mathrm{c}^{\circ}$ )

2) Synthesis of 2-amino-3-cyano-7-hydroxy-4-phenyl (4-chlorophenyl)-4H Chromene D\&E ${ }^{(7-8)}$ (General procedure).

Triethylamine $(0.5 \mathrm{ml})$ was added to a solution of benzaldehyde $(10 \mathrm{mmol})$, malonoitrile $(10 \mathrm{mmol})$ and resorcinol $(10 \mathrm{mmol})$ in ethanol $(30 \mathrm{ml})$. The reaction mixture was refluxed for $0.5 \mathrm{hr}$. The precipitate that formed (2-amino- 3-cyano-7hydroxy -4 -phenyl-4H - Chromene, D and 2-amino-3-cyano-7-hydroxy-4-(4chlorophenyl)-4H-Chromene, E) was filtered off, and crystallized from ethanol .

(Compound D, yield, 63\%, m.p., 209-211 c \& Compound E, yield, 61\%, m.p., $\left.154-156 c^{\circ}\right)$.

3) Synthesis of Dyes I, II, III,IV and V:

The synthesized amino compound (A, B, C, D or E) was diazotized in the usual way by dissolving $(0.1$ mole) of the appropriate amine in hydrochloric acid $(10 \mathrm{ml})$, cooling to $0-5 \mathrm{c}^{\mathrm{o}}$, then adding an equivalent amount of cold sodium nitrite solution with stirring. The cold diazonium salt solution was used for coupling with phenols, amines and compounds containing active methylene group.

The structures and physical data of the synthesized disperse dyes are shown in tables (1 and 2). 


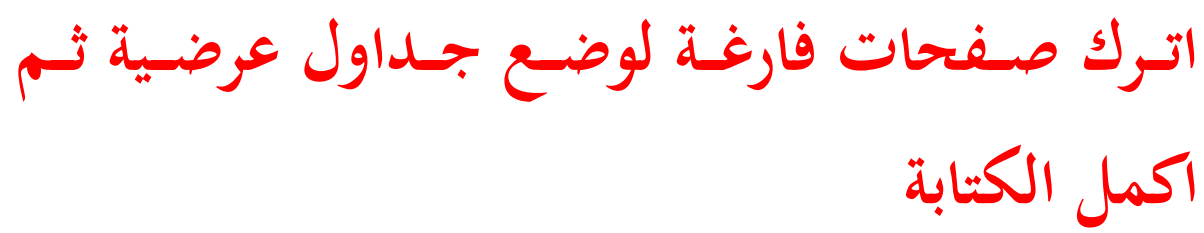


DYEING BEHAVIOR AND PERFORMANCE ... 
S.SHAKRA, et al., 
DYEING BEHAVIOR AND PERFORMANCE ... 
DYEING BEHAVIOR AND PERFORMANCE ... 


\section{II- Materials for dyeing.}

Polyester, Polyester woolen type denir 1.4 and wool produced locally at (Misr Rayon Co. Kafr El Dawer and El Mehalla El Kubra), Egypt.

\section{Instrumentation and measurements.}
1- Melting Point apparatus
2- Infrared spectra
3- Spectrophotometer
4- Elemental analyses

\section{Results and Discussions}

The infrared spectra of parent compounds is basically retained for the reaction products. Some spectral changes are expected to appear as being associated with the formation of new species at the expense of vanished amino group, confirming the appearance of some new characteristic bands for example. 1456 - 1543 corresponding to- $\mathrm{N}=\mathrm{N}$-azo group.

The infrared spectra of dyes I,II, and V which contain the hydroxyl group in ortho position from the azo group show distinct bands in the hydroxyl stretching region. Generally, a series of weak broad bands can be detected in the range 3100 $3420 \mathrm{~cm}^{-1}$ which may be ascribed to a chelate hydrogen- bonded system and the IIIc dye show a band for $\mathrm{CO}$ at 1627.2, this indicate that the dye may be in the hydrazone form.

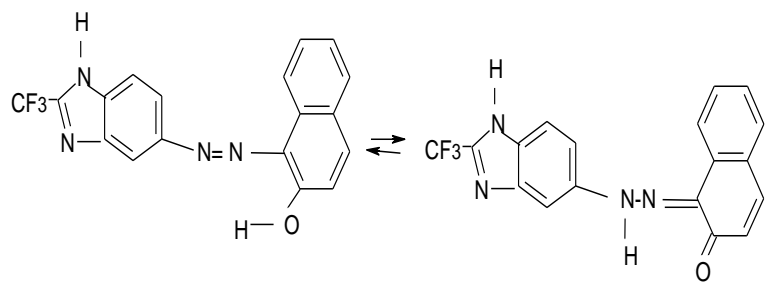

These dyes Ib , IIa,IIb IIIa,IIIb and IVc are not containing hydroxyl group in ortho position to azo group therefor IR, specta show azo group ( $-\mathrm{N}=\mathrm{N}-$ ) bands at $1460-1509$ and also show (NH) band at $3327-3419 \mathrm{~cm}^{-1}$ this suggests that these dyes are predominately in azo form, as opposed to the hydrazo form in the solid state. IR,spectra of dyes Ic, IIc and IIIc are showing a broad band at $3479,3423 \mathrm{~cm}^{-}$ ${ }^{1}$ assigned to $(\mathrm{OH} / \mathrm{NH})$.

The infrared spectra of compounds Ie, IIe, IIIe, IVe show a new band at 3400 $3500 \mathrm{~cm}-1$, respectively, which can be assigned to stretching vibration of $\mathrm{OH}$ group . and this indicates that compound would favour the hydrazone form also, the IR spectra of these compounds show absorption band characteristic of --N=N-stretching at 1557,1543 . Thus, these compounds exist in solid state as a mixture of 
azo - hydrazo form, but for the compound IIe the infrared show the disappearance of $-\mathrm{N}=\mathrm{N}$ - azo group, this indicate that this compound may be found in the hydrazo form.

\subsection{Assessment of prepared derivatives as disperse dyestuffs .}

Table 3 :- UV - visible spectra of prepared dyes in organic solvents .

\begin{tabular}{|c|c|c|c|c|c|c|c|c|c|}
\hline \multirow{3}{*}{$\begin{array}{l}\text { Dye } \\
\text { No }\end{array}$} & \multicolumn{4}{|l|}{\begin{tabular}{|l|} 
Colour in \\
Crystal
\end{tabular}} & \multicolumn{5}{|c|}{ Colour in organic solvents } \\
\hline & & \multicolumn{2}{|c|}{ Ethyl alcohol } & \multicolumn{2}{|l|}{ D M F } & \multirow{2}{*}{\begin{tabular}{|l|}
$\mathrm{CCl}_{4}$ \\
Colour
\end{tabular}} & \multirow{2}{*}{\begin{tabular}{|l} 
Dichloro \\
ethylene
\end{tabular}} & \multirow{2}{*}{$\begin{array}{l}\text { Tetra } \\
\text { chloro } \\
\text { ethylene } \\
\text { colour }\end{array}$} & \multirow{2}{*}{\begin{tabular}{|l|} 
acetone \\
Colour \\
\end{tabular}} \\
\hline & & \begin{tabular}{|l|} 
colour \\
\end{tabular} & $\lambda \max$ & Colour & $\lambda \max$ & & & & \\
\hline I a & $\begin{array}{l}\text { Redish } \\
\text { Brown }\end{array}$ & $\begin{array}{l}\text { deep } \\
\text { orange }\end{array}$ & 421 & $\begin{array}{l}\text { deep } \\
\text { Brown }\end{array}$ & 452 & yellow & yellow & orange & orange \\
\hline $\mathrm{b}$ & $\begin{array}{c}\text { Dark } \\
\text { Brown }\end{array}$ & $\begin{array}{l}\text { Greenish } \\
\text { yellow }\end{array}$ & 436 & $\begin{array}{l}\text { Greenish } \\
\text { yellow }\end{array}$ & 461 & yellow & yellow & G yellow & G yellow \\
\hline $\mathrm{c}$ & $\begin{array}{l}\text { yellowish } \\
\text { Brown }\end{array}$ & $\begin{array}{l}\text { Greenish } \\
\text { orange }\end{array}$ & 474 & Green & 498 & Pale green & Pale green & $\begin{array}{l}\text { Brownish } \\
\text { orange }\end{array}$ & Pale green \\
\hline d & orange & $\begin{array}{l}\text { Light } \\
\text { yellow }\end{array}$ & 429 & yellow & 442 & Pale yellow & $\begin{array}{l}\text { Pale } \\
\text { yellow }\end{array}$ & yellow & yellow \\
\hline $\mathrm{e}$ & Brown & \begin{tabular}{|l|} 
Light \\
yellow
\end{tabular} & 490 & yellow & 515 & Pale yellow & \begin{tabular}{|l} 
Pale \\
yellow
\end{tabular} & Pale yellow & Pale yellow \\
\hline $\mathrm{f}$ & $\begin{array}{l}\text { Brownish } \\
\text { yellow }\end{array}$ & Pale Brown & 414 & Brown & 452 & Pale yellow & $\begin{array}{l}\text { Pale } \\
\text { Brown }\end{array}$ & Pale orange & pale yellow \\
\hline II a & Dark Green & $\begin{array}{l}\text { greenish } \\
\text { yellow }\end{array}$ & 440 & $\begin{array}{l}\text { Greenish } \\
\text { yellow }\end{array}$ & 480 & Pale yellow & $\begin{array}{l}\text { Pale } \\
\text { yellow }\end{array}$ & G yellow & yellow \\
\hline $\mathrm{b}$ & $\begin{array}{l}\text { Greenish } \\
\text { gray }\end{array}$ & Pale yellow & 444 & $\begin{array}{l}\text { Greenish } \\
\text { yellow }\end{array}$ & 482 & Pale Brown & $\begin{array}{l}\text { Pale } \\
\text { Brown }\end{array}$ & \begin{tabular}{|l|}
$\begin{array}{l}\text { Pale } \\
\text { yellow }\end{array}$ \\
\end{tabular} & G yellow \\
\hline $\mathrm{c}$ & $\begin{array}{l}\text { Greenish } \\
\text { orange }\end{array}$ & G.yellow & 485 & G.yellow & 511 & G.yellow & G. yellow & G yellow & G yellow \\
\hline d & orange & Pale yellow & 419 & yellow & 489 & Pale yellow & \begin{tabular}{|l} 
Pale \\
yellow
\end{tabular} & yellow & yellow \\
\hline $\mathrm{e}$ & $\begin{array}{l}\text { orange } \\
\text { greenish }\end{array}$ & \begin{tabular}{|l|}
$\begin{array}{l}\text { Brownish } \\
\text { orange }\end{array}$ \\
\end{tabular} & 498 & \begin{tabular}{|l} 
Brownish \\
orange
\end{tabular} & 520 & Pale orange & $\begin{array}{l}\text { Pale } \\
\text { orange }\end{array}$ & $\begin{array}{l}\text { Brownish } \\
\text { orange }\end{array}$ & \begin{tabular}{|l}
$\begin{array}{l}\text { Brownish } \\
\text { orange }\end{array}$ \\
\end{tabular} \\
\hline $\mathrm{f}$ & $\begin{array}{l}\text { Brownish } \\
\text { yellow }\end{array}$ & $\begin{array}{l}\text { yellowish } \\
\text { Brown }\end{array}$ & 421 & Brown & 452 & $\begin{array}{l}\text { yellowish } \\
\text { Brown }\end{array}$ & $\begin{array}{l}\text { Pale } \\
\text { yellow }\end{array}$ & $\begin{array}{l}\text { yellowish } \\
\text { Brown }\end{array}$ & $\begin{array}{l}\text { yellowish } \\
\text { Brown }\end{array}$ \\
\hline III a & $\begin{array}{l}\text { Redish } \\
\text { Brown } \\
\end{array}$ & \begin{tabular}{|l|} 
deep \\
orange
\end{tabular} & 474 & $\begin{array}{l}\text { deep } \\
\text { orange }\end{array}$ & 498 & Pale yellow & yellow & orange & orange \\
\hline b & \begin{tabular}{|l} 
Brownish \\
green
\end{tabular} & $\begin{array}{l}\text { Greenish } \\
\text { yellow }\end{array}$ & 461 & G yellow & 489 & Pale yellow & $\begin{array}{l}\text { Brownish } \\
\text { yellow }\end{array}$ & $\begin{array}{l}\text { Brownish } \\
\text { yellow }\end{array}$ & $\begin{array}{l}\text { Greenish } \\
\text { yellow }\end{array}$ \\
\hline $\mathrm{c}$ & Dark Grey & G yellow & 481 & \begin{tabular}{|l}
$\begin{array}{l}\text { Brownish } \\
\text { yellow }\end{array}$ \\
\end{tabular} & 509 & - & \begin{tabular}{|l|}
$\begin{array}{l}\text { Greenish } \\
\text { yellow }\end{array}$ \\
\end{tabular} & $\begin{array}{l}\text { Greenish } \\
\text { yellow }\end{array}$ & G yellow \\
\hline d & orange & $\begin{array}{l}\text { deep } \\
\text { yellow }\end{array}$ & 456 & $\begin{array}{l}\text { deep } \\
\text { yellow }\end{array}$ & 482 & Pale yellow & $\begin{array}{l}\text { deep } \\
\text { yellow }\end{array}$ & $\begin{array}{l}\text { deep } \\
\text { yellow }\end{array}$ & Pale yellow \\
\hline $\mathrm{e}$ & orange & Pale yellow & 508 & yellow & 534 & - & yellow & yellow & Pale yellow \\
\hline $\mathrm{f}$ & Brown & \begin{tabular}{|l} 
Brownish \\
yellow
\end{tabular} & 452 & $\begin{array}{l}\text { Brownish } \\
\text { yellow }\end{array}$ & 472 & - & \begin{tabular}{|l|} 
Brownish \\
yellow
\end{tabular} & $\begin{array}{l}\text { Brownish } \\
\text { yellow }\end{array}$ & $\begin{array}{l}\text { Brownish } \\
\text { yellow }\end{array}$ \\
\hline IV a & deep Brown & $\begin{array}{l}\text { Brownish } \\
\text { orange }\end{array}$ & 505 & $\begin{array}{l}\text { Redish } \\
\text { orange }\end{array}$ & 535 & Pale yellow & Pale pink & Pale yellow & $\begin{array}{l}\text { Redish } \\
\text { orange }\end{array}$ \\
\hline b & deep orange & \begin{tabular}{|l|}
$\begin{array}{l}\text { Brownish } \\
\text { orange }\end{array}$ \\
\end{tabular} & 479 & \begin{tabular}{|l} 
Brownish \\
orange
\end{tabular} & 533 & Pale pink & \begin{tabular}{|l} 
Pale \\
Brown \\
\end{tabular} & Pale pink & $\begin{array}{l}\text { Redish } \\
\text { orange }\end{array}$ \\
\hline $\mathrm{c}$ & deep yellow & $\begin{array}{l}\text { deep } \\
\text { orange }\end{array}$ & 437 & \begin{tabular}{|l|} 
deep \\
orange
\end{tabular} & 474 & Pale pink & $\begin{array}{l}\text { deep } \\
\text { orange }\end{array}$ & $\begin{array}{l}\text { deep } \\
\text { orange }\end{array}$ & orange \\
\hline d & deep yellow & $\begin{array}{l}\text { deep } \\
\text { yellow }\end{array}$ & 479 & $\begin{array}{l}\text { deep } \\
\text { yellow }\end{array}$ & 530 & Pale yellow & $\begin{array}{l}\text { Light } \\
\text { yellow }\end{array}$ & $\begin{array}{l}\text { Light } \\
\text { yellow }\end{array}$ & orange \\
\hline $\mathrm{e}$ & \begin{tabular}{|l|} 
yellowish \\
orange
\end{tabular} & $\begin{array}{l}\text { deep } \\
\text { yellow }\end{array}$ & 479 & orange & 533 & - & yellow & yellow & yellow \\
\hline $\mathrm{f}$ & $\begin{array}{l}\text { Redish } \\
\text { Brown }\end{array}$ & $\begin{array}{l}\text { Redish } \\
\text { Brown }\end{array}$ & 515 & $\begin{array}{l}\text { Redish } \\
\text { Brown }\end{array}$ & 534 & - & \begin{tabular}{|l|} 
Brownish \\
orange
\end{tabular} & $\begin{array}{l}\text { Redish } \\
\text { Brown }\end{array}$ & $\begin{array}{l}\text { Brownish } \\
\text { orange }\end{array}$ \\
\hline $\mathrm{g}$ & $\begin{array}{l}\text { Redish } \\
\text { Brown }\end{array}$ & $\begin{array}{l}\text { Redish } \\
\text { Brown }\end{array}$ & 518 & $\begin{array}{l}\text { Redish } \\
\text { Brown }\end{array}$ & 535 & Brown & $\begin{array}{l}\text { Brownish } \\
\text { orange }\end{array}$ & $\begin{array}{l}\text { Redish } \\
\text { Brown }\end{array}$ & $\begin{array}{l}\text { Brownish } \\
\text { orange }\end{array}$ \\
\hline
\end{tabular}


Table 3 Cont:- UV - visible spectra of prepared dyes in organic solvents .

\begin{tabular}{|c|c|c|c|c|c|c|c|c|c|}
\hline \multirow{3}{*}{$\begin{array}{l}\text { Dye } \\
\text { No }\end{array}$} & \multicolumn{4}{|l|}{$\begin{array}{l}\text { Colour in } \\
\text { Crystal }\end{array}$} & \multicolumn{5}{|c|}{ Colour in organic solvents } \\
\hline & & \multicolumn{2}{|c|}{ Ethyl alcohol } & \multicolumn{2}{|l|}{ D M F } & \multirow{2}{*}{\begin{tabular}{|l|}
$\mathrm{CC} \mathrm{I}_{4}$ \\
Colour \\
\end{tabular}} & \multirow{2}{*}{$\begin{array}{l}\begin{array}{l}\text { Dichloro } \\
\text { ethylene }\end{array} \\
\text { Colour } \\
\end{array}$} & \multirow{2}{*}{\begin{tabular}{|l|}
$\begin{array}{l}\text { Tetra } \\
\text { chloro } \\
\text { ethylene }\end{array}$ \\
colour \\
\end{tabular}} & \multirow{2}{*}{$\begin{array}{l}\text { acetone } \\
\text { Colour }\end{array}$} \\
\hline & & colour & $\lambda \max$ & Colour & $\lambda \max$ & & & & \\
\hline h & deep yellow & \begin{tabular}{|l|} 
deep \\
yellow
\end{tabular} & 484 & \begin{tabular}{|l|} 
deep \\
yellow
\end{tabular} & 535 & yellow & yellow & yellow & orange \\
\hline i & \begin{tabular}{|l|} 
yellowish \\
orange
\end{tabular} & $\begin{array}{l}\text { yellowish } \\
\text { orange }\end{array}$ & 488 & orange & 535 & $\begin{array}{l}\text { yellowish } \\
\text { orange }\end{array}$ & yellow & yellow & orange \\
\hline $\mathbf{V} \mathbf{a}$ & Dark Brown & Brown & 503 & Brown & 505 & $\begin{array}{l}\text { Orangish } \\
\text { Brown }\end{array}$ & Brown & Brown & $\begin{array}{l}\text { Brownish } \\
\text { orange }\end{array}$ \\
\hline b & Brown & Brown & 498 & Brown & 504 & $\begin{array}{l}\text { Orangish } \\
\text { Brown }\end{array}$ & Brown & Brown & $\begin{array}{l}\text { Brownish } \\
\text { orange }\end{array}$ \\
\hline c & Brown & Brown & 495 & Brown & 502 & $\begin{array}{l}\text { Orangish } \\
\text { Brown }\end{array}$ & Brown & Brown & $\begin{array}{l}\text { Brownish } \\
\text { orange }\end{array}$ \\
\hline d & \begin{tabular}{|l|} 
yellowish \\
orange
\end{tabular} & $\begin{array}{l}\text { yellowish } \\
\text { orange }\end{array}$ & 486 & orange & 530 & \begin{tabular}{|l|} 
yellowish \\
orange
\end{tabular} & yellow & yellow & orange \\
\hline $\mathrm{e}$ & deep yellow & \begin{tabular}{|l|} 
deep \\
yellow
\end{tabular} & 480 & $\begin{array}{l}\text { deep } \\
\text { yellow }\end{array}$ & 530 & yellow & yellow & yellow & Orange \\
\hline $\mathrm{f}$ & Brown & Brown & 488 & Brown & 498 & $\begin{array}{l}\text { Orangish } \\
\text { Brown }\end{array}$ & Brown & Brown & $\begin{array}{l}\text { Brownish } \\
\text { orange }\end{array}$ \\
\hline
\end{tabular}

The visible spectra of the azo compounds under investigation show one band within the wavelength range $421-535$, this band would be characteristic of $-\mathrm{N}=\mathrm{N}$ - system .

The tautomeric structures of some dyes show a charge transfer from electron donating group to azo group. This electronic migration for dyes to from hydrazo form . ${ }^{(9)}$.

The tatuomeric structures of the investigated dyes ( Ic, IIc, IIIc) show a charge transfer from oxygen atom of the hydroxyl group to the azo group. This electronic migration for dyes to form hydrazo form. ${ }^{(10)}$

\section{From the obtained results it is clear that:-}

The introduction of some substituents on the dye molecule causes some shifts in the position as well as the intensity of the absorption band for example;-

i) The absorption spectrum of dye Ia show band at $\lambda$ max. $421 \mathrm{~nm}$. introduction of acetyl group (electron withdrawing group) in dye $\mathrm{I}_{\mathrm{f}}$ causes a decrease in $\lambda$ max. From $421 \mathrm{n}$ m to $414.0 \mathrm{~nm}$. ( Blue shift ) .

The presence of any substituent group is important. Either to enhance or inhibit this electronic migration thus causing a bathochromic or a hypsochromic shiftsThis can be discussed as follow.

ii) Bathochromic shift may be occurred due to increase conjugation in the parent compounds e.g dye No IIIe. 
ii) The visible spectra of $\mathrm{I}_{\mathrm{b}}, \mathrm{II}_{\mathrm{b}}$ and $\mathrm{III}_{\mathrm{b}}$, show an absorption band for dye $\mathrm{II}_{\mathrm{b}}$ at $444 \mathrm{~nm}$. and absorption band for dye $\mathrm{III}_{\mathrm{b}}$ at $461 \mathrm{~nm}$. This means that a bathochromic shift occurred for dye IIb and IIIb as compared with dye Ib and this is attributed to the increased conjugation in the parent compound.

iii) It was noticed that the increase in the bathochromic shift was due to increase in polarity of solvent and dipole moment of the organic solvent e.g ethanol and carbon tetrachloride.

iv) The visible spectra of dyesIV show that :-

The introduction of electron withdonating group in the coupling component causes a bathochromic effect for dyes IVe and IVf .

v) The effect of hydroxyl or nitro groups in ortho position is more than its effect of hydroxyl or nitro groups in para position e.g dye Ib and Ic and IVh and IVi respectively.

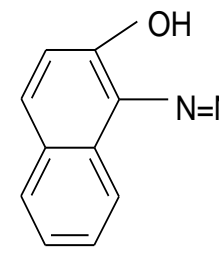<smiles>Cc1ccc2[nH]c(C)nc2c1</smiles>

IIc dye

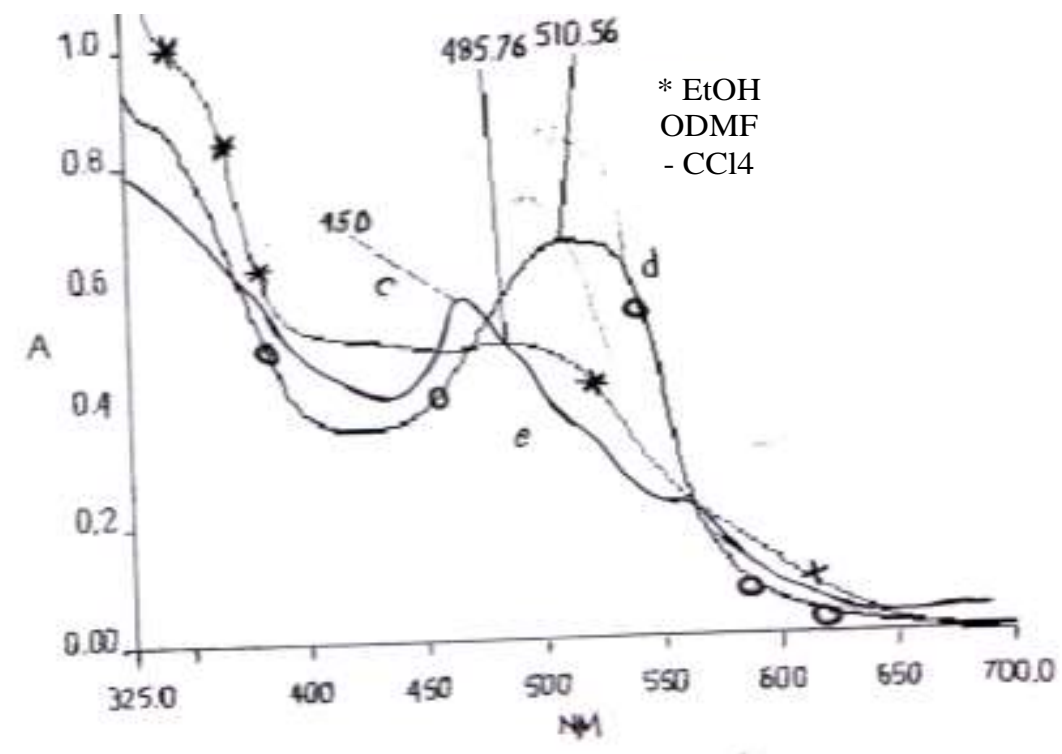

Absorption spectra of dye IIc

Fig., (1) 


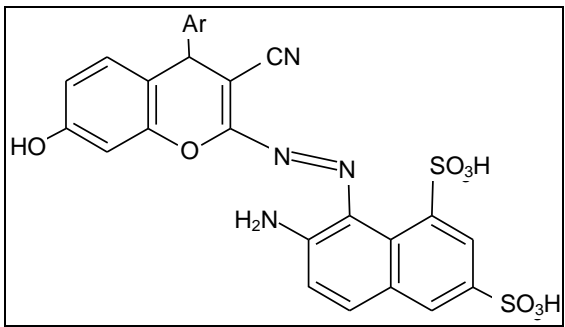

IVa dye

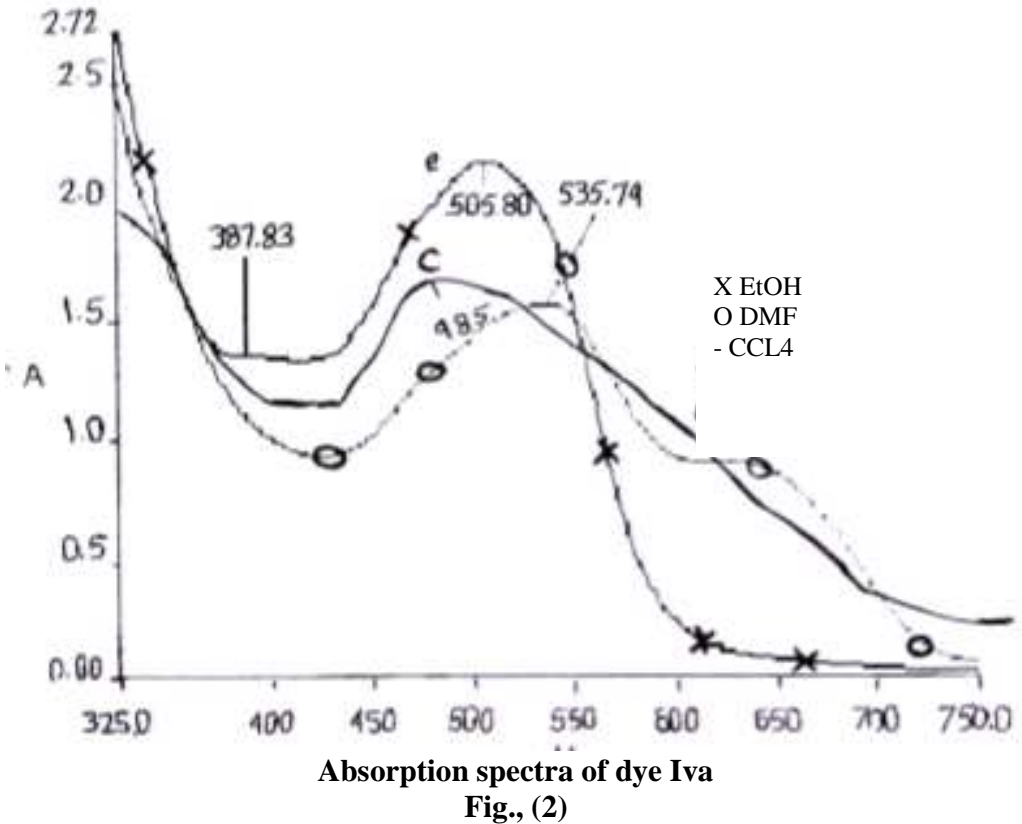

\subsection{Application of the Prepared disperses dyes.}

It was observed visual colour with different shades varied from yellow. Orange to dark brown for disperses dyes under investigation. The coupling component seemed to affect noticeably the depth and colour shade ${ }^{(11)}$.

A range of bright and leveled shade have been obtained on application of these dyes to polyester, polyester woolen type and wool fibre ${ }^{(12)}$.

\subsubsection{Assessment of colour measurement.}

In the present study, the colour strength expressed as K/S values was measured by applying the Kubelka- Munk equation as follow:

$$
\mathrm{K} / \mathrm{S}=\frac{(1-\mathrm{R})^{2}}{2 \mathrm{R}}-\frac{(1-\mathrm{Ro})^{2}}{2 \mathrm{Ro}}
$$

The K/M function has been tested for several prepared dyes on various substrates including polyester, polyester/ wool and wool. As we have seen from tables (4-7) the fibre with increased strength of colour shows low reflection $\mathrm{R} \%$ value but higher $\mathrm{K} / \mathrm{S}$ value indicating darker shade on dyed fibre ${ }^{(13)}$. 
Table(4): - Colour measurement of the dyed polyester fabric samples .

\begin{tabular}{|l|l|l|l|l|l|l|l|l|}
\hline \multirow{2}{*}{$\begin{array}{l}\text { Dye } \\
\text { No. }\end{array}$} & Visual colour & \multicolumn{2}{|l|}{ Measured colour on PET . fibre . } \\
\cline { 2 - 9 } I a & Pale brown & 51.7 & 24.2 & 24.7 & 34.6 & 46.0 & 18 & 2.3 \\
\hline b & Brown & 50.6 & 16.5 & 28.9 & 32.5 & 33.9 & 20 & 2.5 \\
\hline c & Orange & 62.3 & 24.6 & 16.5 & 28.0 & 79.2 & 18 & 2.3 \\
\hline d & Deep yellow & 50.9 & 15.6 & 19.3 & 24.8 & 51.1 & 18 & 2.4 \\
\hline e & light yellow & 64.9 & 3.1 & 13.6 & 13.9 & 47.0 & 22 & 1.3 \\
\hline f & $\begin{array}{l}\text { Yellowish } \\
\text { orange }\end{array}$ & 61.7 & 24.2 & 24.7 & 34.6 & 46.0 & 18 & 2.9 \\
\hline II a & Deep yellow & 64.9 & 3.2 & 15.9 & 15.9 & 60.2 & 30 & 1.9 \\
\hline b & $\begin{array}{l}\text { Orangish } \\
\text { Brown }\end{array}$ & 56.7 & 17.1 & 27.2 & 32.3 & 58.1 & 31 & 3.7 \\
\hline c & Deep orange & 50.1 & 17.8 & 18.7 & 14.8 & 35.6 & 18 & 2.1 \\
\hline d & $\begin{array}{l}\text { Yellowish } \\
\text { orange }\end{array}$ & 50.1 & 12.0 & 14.0 & 15.6 & 38.9 & 16 & 2.8 \\
\hline e & Yellow & 63.1 & 26.2 & 26.7 & 35.6 & 41.2 & 35 & 1.6 \\
\hline f & Yellow & 50.9 & 15.6 & 19.3 & 24.8 & 51.1 & 18 & 2.4 \\
\hline III a & Orange & 50.9 & 30.39 & 20.1 & 35.9 & 32.1 & 13 & 3.3 \\
\hline b & $\begin{array}{l}\text { Yellowish } \\
\text { Brown }\end{array}$ & 62.9 & 19.1 & 28.1 & 32.1 & 58.1 & 37 & 3.6 \\
\hline c & Deep orange & 50.1 & 17.8 & 18.7 & 14.8 & 35.6 & 18 & 2.3 \\
\hline d & $\begin{array}{l}\text { Yellowish } \\
\text { orange }\end{array}$ & 50.1 & 12.0 & 14.0 & 15.6 & 38.9 & 16 & 2.8 \\
\hline e & Yellow & 61.7 & 24.2 & 24.7 & 34.6 & 46.0 & 18 & 1.9 \\
\hline f & Deep Orange & 50.9 & 30.39 & 20.1 & 35.9 & 32.1 & 13 & 3.3 \\
\hline Iv a & Pale Brown & 56.72 & 17.01 & 27.13 & 32.02 & 57.91 & 11 & 3.7 \\
\hline b & Pale orange & 49.98 & 5.4 & 9.0 & 10.5 & 59.2 & 15 & 2.8 \\
\hline c & Deep yellow & 69.82 & 14.7 & 19.3 & 24.8 & 52.1 & 18 & 2.5 \\
\hline d & Dark Yellow & 64.9 & 3.09 & 13.6 & 13.88 & 77.3 & 30 & 1.6 \\
\hline e & yellow & 65.8 & 3.1 & 13.5 & 13.80 & 77.1 & 35 & 1.9 \\
\hline f & Brown & 44.5 & 10.5 & 4.76 & 11.53 & 24.4 & 9 & 3.3 \\
\hline
\end{tabular}

Table(5): Colour measurement of the dyed wool fabric samples .

\begin{tabular}{|c|c|c|c|c|c|c|c|c|}
\hline \multirow{2}{*}{$\begin{array}{l}\text { Dye } \\
\text { No. }\end{array}$} & \multirow{2}{*}{$\begin{array}{l}\text { Visual } \\
\text { colour on } \\
\text { Wool }\end{array}$} & \multicolumn{7}{|c|}{ Measured colour on wool . fibre } \\
\hline & & $\mathbf{L}^{*}$ & $\mathbf{a}^{*}$ & $\mathbf{b}^{*}$ & $\mathbf{C}^{*}$ & H & $\mathbf{R}$ & $\mathbf{K} / \mathbf{S}$ \\
\hline III a & Brown & 52.7 & 32.2 & 27.1 & 31.9 & 57.9 & 20 & 4.3 \\
\hline $\mathrm{b}$ & $\begin{array}{l}\text { Deep } \\
\text { Brown }\end{array}$ & 44.55 & 10.5 & 4.76 & 11.6 & 24.4 & 10 & 6.3 \\
\hline $\mathrm{c}$ & Pale Brown & 49.86 & 7.5 & 9.6 & 12.4 & 34.2 & 28 & 3.13 \\
\hline $\mathrm{d}$ & $\begin{array}{l}\text { Deep } \\
\text { yellow }\end{array}$ & 62.6 & 36.8 & 43.7 & 57.1 & 49.9 & 22 & 3.9 \\
\hline $\mathrm{e}$ & $\begin{array}{l}\text { Pale } \\
\text { Orange }\end{array}$ & 50.9 & 30.5 & 20.3 & 35.9 & 32.1 & 28 & 3.8 \\
\hline $\mathrm{f}$ & $\begin{array}{l}\text { Pale } \\
\text { Orange }\end{array}$ & 50.6 & 16.5 & 28.9 & 32.5 & 33.9 & 20 & 4.5 \\
\hline
\end{tabular}


Table (6) : Colour measurement on Polyester/ wool:

\begin{tabular}{|l|l|l|l|l|l|l|l|l|}
\hline \multirow{2}{*}{$\begin{array}{l}\text { Dye } \\
\text { No }\end{array}$} & Visual colour & \multicolumn{8}{|l|}{ Measured Colour on PET/ wool fibre } \\
\cline { 3 - 10 } & P.E. $/$ wool & $\mathbf{L}^{*}$ & $\mathbf{a}^{*}$ & $\mathbf{b}^{*}$ & $\mathbf{C}$ & $\mathbf{H}$ & $\mathbf{R}$ & $\mathbf{k} / \mathbf{S}$ \\
\hline $\mathrm{I}_{\mathrm{f}}$ & Deep Yellow & 61.7 & 24.2 & 24.7 & 34.6 & 46.0 & 18 & 3.1 \\
\hline $\mathrm{II}_{\mathrm{f}}$ & Deep yellow & 50.9 & 15.6 & 19.3 & 24.8 & 51.1 & 18 & 2.9 \\
\hline $\mathrm{III}_{\mathrm{f}}$ & Deep Orange & 50.9 & 30.39 & 20.1 & 35.9 & 32.1 & 13 & 3.8 \\
\hline $\mathrm{IV}_{\mathrm{d}}$ & Deep yellow & 69.82 & 14.7 & 19.3 & 24.8 & 52.1 & 18 & 2.9 \\
\hline $\mathrm{IV}_{\mathrm{e}}$ & Yellow & 65.8 & 3.1 & 13.5 & 13.8 & 77.1 & 35 & 1.9 \\
\hline
\end{tabular}

Table (7) : Colour measurement on wool fibre:

\begin{tabular}{|c|c|c|c|c|c|c|c|c|}
\hline \multirow{2}{*}{$\begin{array}{l}\text { Dye } \\
\text { No }\end{array}$} & \multirow{2}{*}{$\begin{array}{l}\text { Visual colour } \\
\text { on Wool }\end{array}$} & \multicolumn{7}{|c|}{ Measured Colour on Wool fibre } \\
\hline & & $\mathbf{L}^{*}$ & $\mathbf{a}^{*}$ & $\mathbf{b}^{*}$ & $\mathbf{C}^{*}$ & $\mathbf{H}$ & $\mathbf{R}$ & $\mathbf{k} / \mathbf{S}$ \\
\hline $\mathrm{I}_{\mathrm{f}}$ & Orange & 50.6 & 16.5 & 28.9 & 32.5 & 33.9 & 24 & 4.3 \\
\hline $\mathrm{II}_{\mathrm{f}}$ & Orange & 50.9 & 30.39 & 20.1 & 35.9 & 32.1 & 28 & 3.8 \\
\hline $\mathrm{III}_{\mathrm{f}}$ & Pale Brown & 56.7 & 16.98 & 27.1 & 31.9 & 57.9 & 18 & 4.5 \\
\hline $\mathrm{IV}_{\mathrm{d}}$ & Yellow & 63.9 & 38.95 & 46.23 & 60.4 & 49.8 & 22 & 3.9 \\
\hline $\mathrm{IV}_{\mathrm{e}}$ & Yellow & 62.6 & 36.8 & 43.75 & 57.1 & 49.9 & 22 & 3.9 \\
\hline
\end{tabular}

From the above results the K/S values for various dyed substrate are in the following order. Wool > polyester/wool > polyester.

\section{Conclusion}

In this research we could prepared disperse dyes from benzimidazole and chromene applied to dye polyester, polyester woolen type and wool.A range of bright and leveled shade have been obtained on application of these disperse dyes. From the above results the K/S values for various dyed substrate are in the following order, Wool > polyester/wool > polyester.

\section{References :}

1. Y.,Li, Y.,Tang, S.,Zhang, and I., Yang, Text., Res. J. 77(9) , 703-705,(2007).

2. M.A., Rahman , S., Shakra , A.A., Ghattas , and H.M., Mostafa, Am ., dye , Repo ., (1994) .

3. M.M.,Ramla , M. A., Omar , H., Tokuda, and H. I., El Diwain , Bioorg . Med . chem. ., 15 , 6489-6492, (2007) .

4. G. K., Desai., and R.K Desai, J. Saudi chem., Soc ., 9,631 - 460 , (2006) .

5. G.K., Desai , and R. K., Desai , J. Sulfur chem.,27, 315 -38, (2006) .

6. G. K ., Desai, and R. K., Desai, Bioorg . Med . chem., 14, $8271-8279$, (2006) .

7. A.M., Shestopalov, Yu.M., Emelianova, and V.N., Nesterov, Russ., Chem.., Bull., 51 (12),2238- 2243,(2002).

8. M.M., Khafagy , A.H., EL- wahas , F.A., Eid, and A.M ., Agrody, farmco., 57,715-722 ,( 2002) .

9. R. M., Silverstein, F.X .,Webster . Spectrometric identification of organic compounds . New York : John Wiley \& Sons, ( 1998) .

10. M.S.,Yen, and I.J., wang, Dyes and pigments.,61,243 , (2004) .

11. M.S., Yen, and I.J.,Wang, Dyed and Pigments 67,183 - 188, (2004) .

12. M.S., Yen, and I.J.,wang, Dyes and Pigments $62,173-180$, (2004)..

13. Y.,Li, Y.,Tang, S.,Zhang, and I., Yang, Text., Res. J. 77(9) , 703-709,(2007). 
DYEING BEHAVIOR AND PERFORMANCE ... 

Al-Azhar Bull. Sci. Vol. 24, No. 1 (June.): pp. 125-140, 2013. 
Table 1: physical data of disperse dyes derived from 5-aminobenzimidazole derivatives I- III.

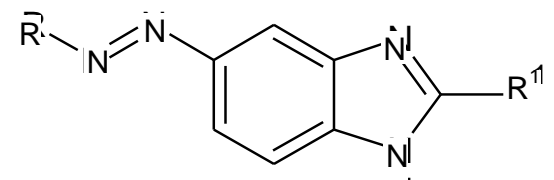

$\mathrm{HI}$

I - III

I, $\mathrm{R}^{1}=\mathrm{H} ; \mathrm{II}, \mathrm{R}^{1}=\mathrm{CH}_{3} ; \mathrm{III}, \mathrm{R}^{1}=\mathrm{CF}_{3}$.

\begin{tabular}{|c|c|c|c|c|c|c|c|c|c|}
\hline $\begin{array}{l}\text { Dye } \\
\text { No }\end{array}$ & $\mathrm{R}$ & $\begin{array}{c}\text { Yield } \\
\%\end{array}$ & $\mathbf{m} \cdot \mathbf{p}^{\circ} \mathbf{C}$ & $\begin{array}{l}\text { M.formula } \\
\text { (M.W.t) }\end{array}$ & $\mathrm{C}^{(\mathrm{c}}$ & $\begin{array}{l}\text { lemen } \\
\text { analys } \\
\text { l/ four } \\
\text { H }\end{array}$ & & $\operatorname{IR}\left(v, \mathrm{~cm}^{-1}\right)$ & $\begin{array}{l}\text { Colour } \\
\text { of solid }\end{array}$ \\
\hline Ia & & 74 & $>300$ & $\begin{array}{l}\mathrm{C}_{13} \mathrm{H}_{11} \mathrm{~N}_{5} \mathrm{O} \\
\quad(253)\end{array}$ & $\begin{array}{l}61.66 \\
61.60\end{array}$ & $\begin{array}{l}4.35 \\
4.31\end{array}$ & $\begin{array}{l}27.67 \\
27.62\end{array}$ & $\begin{array}{l}\text { IR:3416.3(OH/NH } / \mathrm{NH}) \text { broad;2928.3(CH); } \\
1595.3(\mathrm{C}=\mathrm{N}), 1395.7(\mathrm{C}-\mathrm{N}) 1460 \quad(\mathrm{~N}=\mathrm{N}) .\end{array}$ & $\begin{array}{l}\text { Redish } \\
\text { Brown }\end{array}$ \\
\hline $\mathrm{Ib}$ & & 90 & $>300$ & $\begin{array}{l}\mathrm{C}_{17} \mathrm{H}_{12} \mathrm{~N}_{4} \mathrm{O} \\
\quad(288)\end{array}$ & $\begin{array}{l}70.83 \\
70.80\end{array}$ & $\begin{array}{l}4.16 \\
4.10\end{array}$ & $\begin{array}{l}19.44 \\
19.40\end{array}$ & $\begin{array}{c}\text { IR: } 3417.3(\mathrm{OH}, \mathrm{NH}) \text { broad;2930.2(CH) } \\
\text { 1383.1(C- N), } 1457.4(\mathrm{~N}=\mathrm{N})\end{array}$ & $\begin{array}{l}\text { Dark } \\
\text { Brown }\end{array}$ \\
\hline Ic & & 76 & $\begin{array}{l}210 \\
\text { decom. }\end{array}$ & $\begin{array}{l}\mathrm{C}_{17} \mathrm{H}_{12} \mathrm{~N}_{4} \mathrm{O} \\
\quad(288)\end{array}$ & $\begin{array}{l}70.83 \\
70.70\end{array}$ & $\begin{array}{l}4.16 \\
4.08\end{array}$ & $\begin{array}{l}19.44 \\
19.42\end{array}$ & 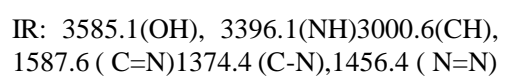 & $\begin{array}{l}\text { yellowish } \\
\text { Brown }\end{array}$ \\
\hline Id & & 94 & $>300$ & $\begin{array}{l}\mathrm{C}_{21} \mathrm{H}_{14} \mathrm{~N}_{4} \mathrm{O} \\
\quad(338)\end{array}$ & $\begin{array}{l}74.56 \\
74.50\end{array}$ & $\begin{array}{l}4.14 \\
4.10\end{array}$ & $\begin{array}{l}16.57 \\
16.50\end{array}$ & $\begin{array}{l}\text { IR: } 3469.4(\mathrm{OH}), 3417.3(\mathrm{NH}), \quad 2926.4 \\
(\mathrm{CH}), 1638.7(\mathrm{C}=\mathrm{O}), \quad 1460.3(\mathrm{~N}=\mathrm{N}) .\end{array}$ & $\begin{array}{c}\text { Brownish } \\
\text { orange }\end{array}$ \\
\hline
\end{tabular}


Table 1:(Cont.),

\begin{tabular}{|c|c|c|c|c|c|c|c|}
\hline Ie & 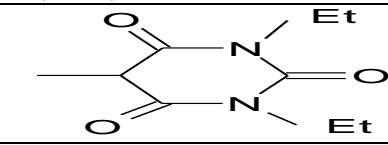 & 80 & $292+4$ & $\begin{array}{c}\mathrm{C}_{15} \mathrm{H}_{16} \mathrm{~N}_{6} \mathrm{O}_{3} \\
(328)\end{array}$ & $\begin{array}{lll}54.88 & 4.88 & 25.60 \\
54.80 & 4.80 & 25.60\end{array}$ & $\begin{array}{l}\mathrm{IR}: 3511.8(\mathrm{OH}), 3321.8,3239.8 \quad(\mathrm{NH}) \\
, 1630(\mathrm{C}=0), 1380.2(\mathrm{C}-\mathrm{N}), \quad 1557.7 \\
(\mathrm{C}=\mathrm{N}),(\mathrm{N}=\mathrm{N})\end{array}$ & Brown \\
\hline If & $\left.\right|_{\mathrm{CL}} ^{\mathrm{CH}}$ & 78 & $\begin{array}{c}220 \\
\text { decom }\end{array}$ & $\begin{array}{c}\mathrm{C}_{15} \mathrm{H}_{12} \mathrm{~N}_{5} \mathrm{O}_{2} \mathrm{Cl} \\
(329.5)\end{array}$ & $\begin{array}{ccc}54.63 & 3.64 & 1.24 \\
54.55 & 3.60 & 1.20\end{array}$ & $\begin{array}{l}\text { IR: } 3482.9(\mathrm{OH}, \mathrm{NH}) \text { broad;2932.2868.2 } \\
(\mathrm{CH} . \text { aliph }) ; 1671.5(\mathrm{C}=0) ; 1498.9(-\mathrm{N}=\mathrm{N}- \\
\text { ); :868.1( C- CL) }\end{array}$ & $\begin{array}{l}\text { Brownish } \\
\text { gray }\end{array}$ \\
\hline IIa & & 90 & $>300$ & $\begin{array}{c}\mathrm{C}_{14} \mathrm{H}_{13} \mathrm{~N}_{5} \mathrm{O} \\
(267)\end{array}$ & 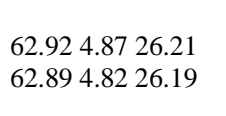 & $\begin{array}{l}\text { IR : } 3419.2\left(\mathrm{OH}, \mathrm{NH}_{2}, \mathrm{NH}\right) \text { broad, } \\
2932.2(\mathrm{CH}), 1509.51(\mathrm{~N}=\mathrm{N}) \\
1374.4(\mathrm{C}-\mathrm{N})\end{array}$ & $\begin{array}{l}\text { Dark } \\
\text { Brown }\end{array}$ \\
\hline IIb & & 94 & $>300$ & $\begin{array}{c}\mathrm{C}_{18} \mathrm{H}_{14} \mathrm{~N}_{4} \mathrm{O} \\
\quad(302)\end{array}$ & $\begin{array}{lll}71.52 & 4.64 & 18.54 \\
71.50 & 4.60 & 18.50\end{array}$ & $\begin{array}{l}\text { IR: } 3327.6(\mathrm{OH}, \mathrm{NH}) \text { broad , } 3055.6 \\
(\mathrm{CH}), 1586.6(\mathrm{C}=\mathrm{N}), 1371.6(\mathrm{C}-\mathrm{N}), \\
1517.2(\mathrm{~N}=\mathrm{N}) .) .\end{array}$ & $\begin{array}{l}\text { Brownish } \\
\text { gray }\end{array}$ \\
\hline IIc & & 96 & $>300$ & $\begin{array}{c}\mathrm{C}_{18} \mathrm{H}_{14} \mathrm{~N}_{4} \mathrm{O} \\
(302)\end{array}$ & $\begin{array}{lll}71.52 & 4.64 & 18.54 \\
71.50 & 4.60 & 18.49\end{array}$ & $\begin{array}{l}\text { IR: } 3479(\mathrm{OH}), 3399(\mathrm{NH}), 3050.8( \\
\mathrm{CH}), 1599.2(\mathrm{C}=\mathrm{N}), 1378.3(\mathrm{C}-\mathrm{N}) \\
1466.1 \\
(\mathrm{~N}=\mathrm{N})\end{array}$ & $\begin{array}{l}\text { Brownish } \\
\text { orange }\end{array}$ \\
\hline IId & & 88 & $\begin{array}{c}280 \\
\text { Decomp. }\end{array}$ & $\begin{array}{c}\mathrm{C}_{22} \mathrm{H}_{16} \mathrm{~N}_{4} \mathrm{O} \\
(352)\end{array}$ & $\begin{array}{lll}75.0 & 4.55 & 15.90 \\
75.0 & 4.49 & 15.85\end{array}$ & $\begin{array}{l}\text { IR: } 3405.7(\mathrm{OH} / \mathrm{NH}) \text { broad, } 1652.2(\mathrm{C}=\mathrm{O}) \\
1596.3(\mathrm{C}=\mathrm{N}), 1382.2 \quad(\mathrm{C}-\mathrm{N}) \\
1466.1(\mathrm{~N}=\mathrm{N}) .)\end{array}$ & $\begin{array}{l}\text { Orangeish } \\
\text { Brown }\end{array}$ \\
\hline IIe & Et & 92 & $\begin{array}{c}280 \\
\text { Decomp }\end{array}$ & $\begin{array}{c}\mathrm{C}_{16} \mathrm{H}_{18} \mathrm{~N}_{6} \mathrm{O}_{3} \\
\quad(342)\end{array}$ & $\begin{array}{lll}56.14 & 5.26 & 24.56 \\
56.10 & 5.20 & 2452\end{array}$ & $\begin{array}{l}\text { IR : } 3400.0(\mathrm{OH}) /(\mathrm{NH}), 3082.6(\mathrm{CH}), \\
2942.8,28675(\mathrm{CH}), 1763.2,1695 .(2 \\
\mathrm{C}=\mathrm{O}),(\mathrm{C}-\mathrm{N}) 1387.0 .\end{array}$ & $\begin{array}{l}\text { orange } \\
\text { Brownish }\end{array}$ \\
\hline
\end{tabular}


Table 1:(Cont.).

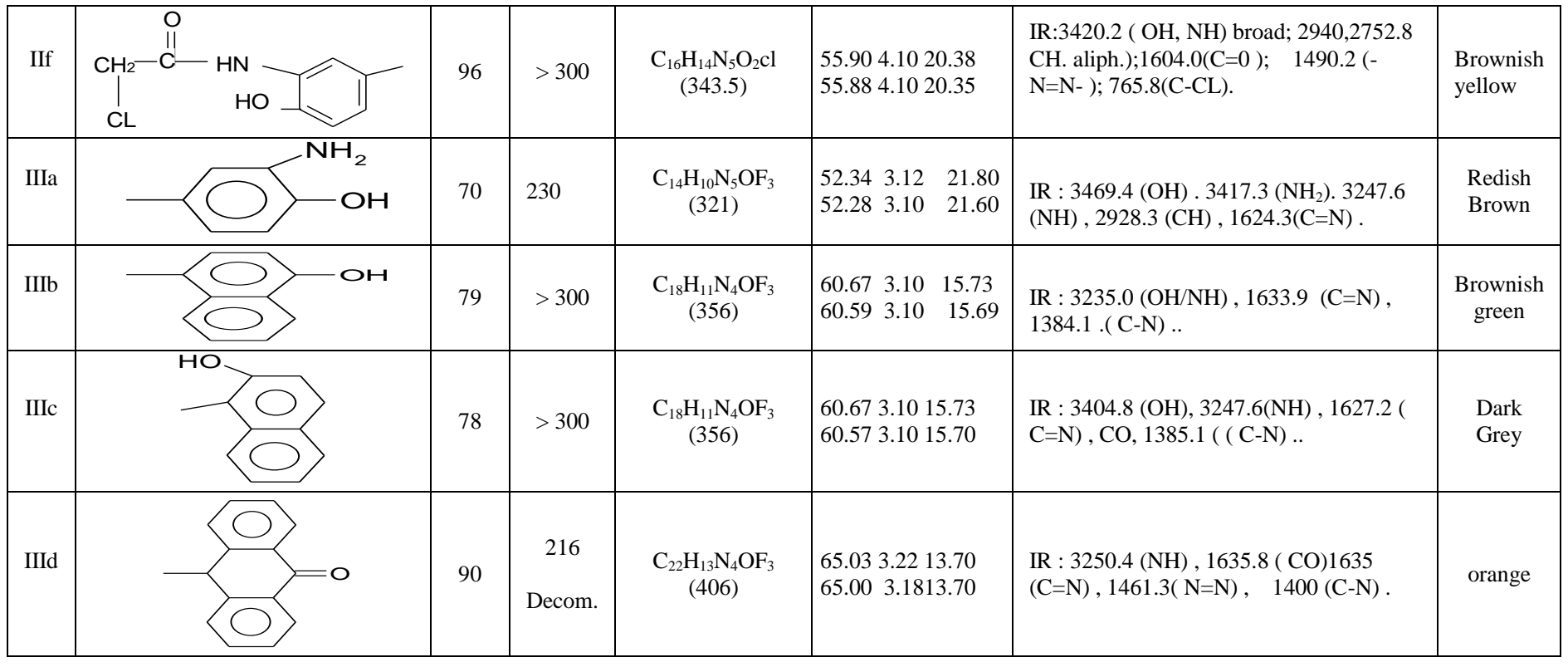


Table 1:(Cont.).

\begin{tabular}{|c|c|c|c|c|c|c|c|}
\hline IIIe & $\sum_{O}^{O} N_{E=t}^{E t}$ & 80 & 284 & $\begin{array}{c}\mathrm{C}_{16} \mathrm{H}_{15} \mathrm{~N}_{6} \mathrm{O}_{3} \mathrm{~F}_{3} \\
\text { (396) }\end{array}$ & $\begin{array}{l}48.483 .79 \\
21.20 \\
48.403 .70 \\
21.18\end{array}$ & $\begin{array}{l}\text { IR } 3435.6(\mathrm{OH}, \mathrm{NH}) \text { broad, } \\
2931.2(\mathrm{CH}), \quad 1658(\mathrm{C}=\mathrm{O}), 1543.2( \\
\mathrm{C}=\mathrm{N}) \quad(\mathrm{N}=\mathrm{N}), 1385.1(\mathrm{C}-\mathrm{N})\end{array}$ & $\begin{array}{c}\text { Brownish } \\
\text { orange }\end{array}$ \\
\hline IIIf & $\left.\right|_{\mathrm{CL}} ^{\mathrm{CH}} \stackrel{\mathrm{O}}{\mathrm{C}}-\mathrm{HO}$ & 80 & $>300$ & $\begin{array}{c}\mathrm{C}_{16} \mathrm{H}_{11} \mathrm{~N}_{5} \mathrm{O}_{2} \mathrm{CLF}_{3} \\
(397.5)\end{array}$ & $\begin{array}{ll}48.30 & 2.7 \\
17.61 & \\
48.20 & 2.7 \\
17.58 & \end{array}$ & $\begin{array}{l}\text { IR :3502.2(OH, NH) broad, } 24932.2 \text {, } \\
2870.4(\mathrm{CH} . \\
\text { aliph):1672.5(C=0);1498.9(-N=N- ); } \\
866.1 \mathrm{C}-\mathrm{CL}\end{array}$ & $\begin{array}{l}\text { Dark } \\
\text { Brown }\end{array}$ \\
\hline
\end{tabular}


Table 2 : physical data of disperse dyes derived from 2-amino- 3-cyano -7-hydroxy -4-aryl -4H-chromene.<smiles>[R]N=NC1=C(C#N)C(Br)c2ccc(O)cc2O1</smiles>

IV \& V

IV, Ar=phenyl \& V,Ar=4-chlorophenyl.

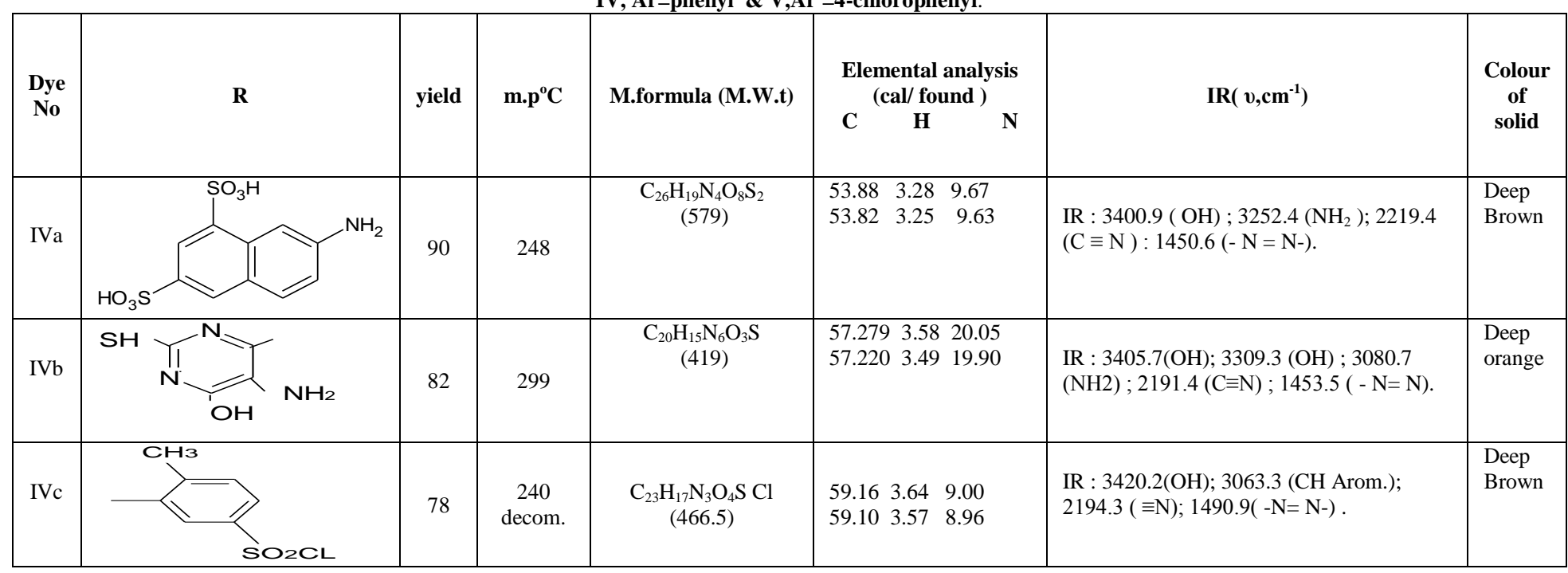


Table 2:(Cont.).

\begin{tabular}{|c|c|c|c|c|c|c|c|c|}
\hline IVd & $\mathrm{NH2}$ & 84 & $\begin{array}{c}200 \\
\text { decom. }\end{array}$ & $\begin{array}{l}\mathrm{C}_{22} \mathrm{H}_{16} \mathrm{~N}_{5} \mathrm{O}_{4} \\
\quad(414)\end{array}$ & \multicolumn{2}{|c|}{$\begin{array}{lll}63.77 & 3.86 & 16.91 \\
63.70 & 3.80 & 16.83\end{array}$} & $\begin{array}{l}\text { IR: } 3427.9(\mathrm{OH}): 3153\left(\mathrm{NH}_{2}\right) ; 2207.8(\mathrm{C} \equiv \\
\mathrm{N}) ; 1598.2,1540.3 \quad \mathrm{NO}_{2}, 1467(-\mathrm{N}=\mathrm{N}-)\end{array}$ & $\begin{array}{l}\text { Deep } \\
\text { Yellow }\end{array}$ \\
\hline IVe & & 53 & 270 & $\begin{array}{l}\mathrm{C}_{22} \mathrm{H}_{15} \mathrm{~N}_{3} \mathrm{O}_{3} \mathrm{Br} \\
\text { (449) }\end{array}$ & \multicolumn{2}{|c|}{$\begin{array}{lll}58.80 & 3.34 & 9.35 \\
58.73 & 3.28 & 9.30\end{array}$} & $\begin{array}{l}\text { IR: } 3424.1,3331.5(2 \mathrm{OH}), 2184.6(\mathrm{C} \equiv \mathrm{N}) \\
1403.4(-\mathrm{N}=\mathrm{N}-)\end{array}$ & $\begin{array}{l}\text { Brownish } \\
\text { yellow }\end{array}$ \\
\hline IVf & & 92 & 235 & $\begin{array}{l}\mathrm{C}_{22} \mathrm{H}_{17} \mathrm{~N}_{4} \mathrm{O}_{3} \\
\quad(385)\end{array}$ & \multicolumn{2}{|c|}{$\begin{array}{lll}68.57 & 4.42 & 14.55 \\
68.49 & 4.40 & 14.48\end{array}$} & $\begin{array}{l}\text { IR: } 3423.1,3334.4(2 \mathrm{OH}), 3223.4 \\
(\mathrm{NH} 2) ; 2188.5 \\
(\mathrm{C} \equiv \mathrm{N}) ; 1402.4(-\mathrm{N}=\mathrm{N}-)\end{array}$ & $\begin{array}{l}\text { Reddish } \\
\text { brown }\end{array}$ \\
\hline $\mathrm{IVg}$ & & 86 & 210 & $\begin{array}{c}\mathrm{C}_{22} \mathrm{H}_{17} \mathrm{~N}_{4} \mathrm{O}_{3} \\
\quad(385)\end{array}$ & $\begin{array}{l}68.57 \\
68.53\end{array}$ & $\begin{array}{l}14.55 \\
14.53\end{array}$ & & $\begin{array}{l}\text { Reddish } \\
\text { brown }\end{array}$ \\
\hline IVh & & 89 & $\begin{array}{c}180 \\
\text { Decomp. }\end{array}$ & $\begin{array}{l}\mathrm{C}_{22} \mathrm{H}_{15} \mathrm{~N}_{6} \mathrm{O}_{6} \\
\quad(459)\end{array}$ & $\begin{array}{ll}57.52 & 3.27 \\
57.51 & 3.25\end{array}$ & $\begin{array}{l}18.30 \\
18.29\end{array}$ & & $\begin{array}{l}\text { Deep } \\
\text { Yellow }\end{array}$ \\
\hline IVi & & 90 & $\begin{array}{c}175 \\
\text { Decomp. }\end{array}$ & $\begin{array}{l}\mathrm{C}_{22} \mathrm{H}_{15} \mathrm{~N}_{6} \mathrm{O}_{6} \\
\quad(459)\end{array}$ & $\begin{array}{ll}57.57 & 3.27 \\
57.51 & 3.23\end{array}$ & $\begin{array}{l}18.30 \\
18.28\end{array}$ & & $\begin{array}{l}\text { Deep } \\
\text { Yellow }\end{array}$ \\
\hline
\end{tabular}


Table 2: (cont.)

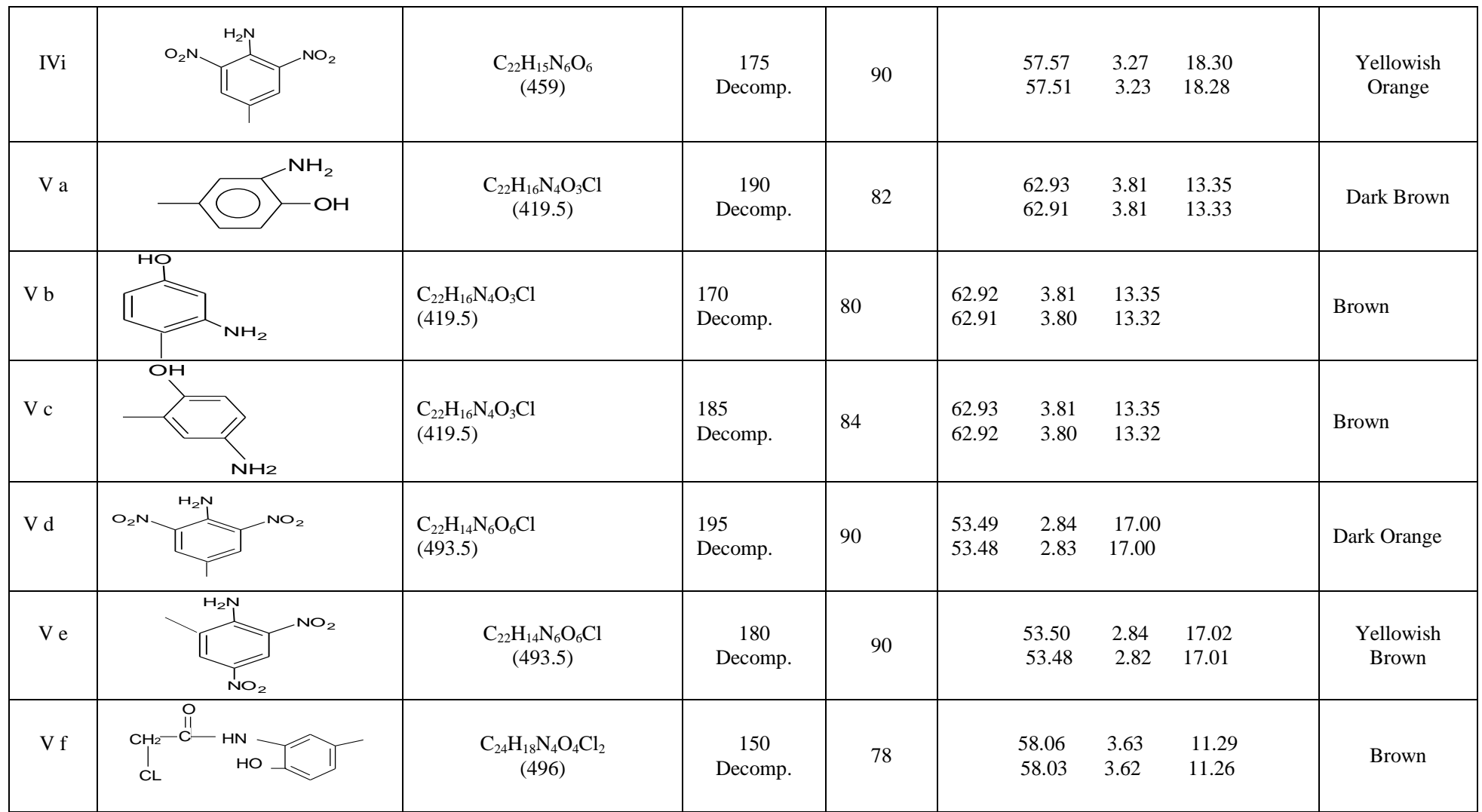


